\title{
Scientific scope of integrating research activities in the Janus Serum Bank and Cancer Registry of Norway
}

\author{
Hilde Langseth, Randi Gislefoss, Jan Ivar Martinsen and Steinar Tretli \\ Cancer Registry of Norway, Institute of Population-Based Cancer Research, Department of Research, Oslo, Norway \\ Correspondence: Hilde Langseth, Cancer Registry of Norway, Institute of Population-Based Cancer Research, Department of Research, \\ P.O. Box 5313 Majorstuen, NO-0304 Oslo, Norway \\ E-mail: hilde.langseth@kreftregisteret.no Telephone: +4722451300 Telefax: + 4722451370
}

\section{BACKGROUND}

In recent years there has been an increased interest in using biorepositories in cancer epidemiology to investigate etiological factors as well as define biomarkers of early detection, treatment and prognosis. Advancing cancer research through biospecimen science requires a high focus on quality $(1,2)$. When introducing molecular epidemiology in the 1980 s, the expectation was that it would help overcome some major limitations of epidemiology and facilitate cancer prevention. This first generation of markers has contributed to the understanding of risk and susceptibility related largely to genotoxic carcinogens. Today a number of new and promising biomarkers are becoming available for epidemiological studies, thanks to the development of high-throughput technologies and theoretical advances in biology (3). One major challenge in biobank-based research is the access to large series of high quality biospecimens and data on disease outcome and confounding variables.

The Janus Serum Bank was established in the early 1970s with the aim of searching in the premorbid sera for chemical, biochemical, immunological, or other changes that might indicate cancer development at early stages, or be indicative of increased risk of cancer (4-6). It is a population-based biobank with blood serum samples from 317000 individuals from 17 counties in Norway. The main part of the collection derives from screening health examination participants (90\%) and the remaining $10 \%$ is from Red Cross blood donors in Oslo. The Norwegian Cancer Society financed the operation of the bank up to 2004, since then it has been fully integrated within the Cancer Registry of Norway. This integration optimizes the use of the biobank by providing the unique opportunity to contribute with biological specimens and high quality clinical data from cancer patients. The development, sample collection, quality assurance and methodological considerations in Janus projects are described elsewhere (7). In the present communication we want to describe the experiences from 35 years of operation of a prospective cancer biobank.

\section{QUALITY ASPECTS IN BIOBANKING}

Good biobanking practice requires a quality manual containing Standard Operational Procedures (SOPs) to ensure professional handling at all levels of operation.
The aim of a quality manual is to specify routines and methods for storage and use of the specimens that will give the best scientific return within the available budget (8). To meet these requirements a Janus quality manual was compiled about ten years ago, as part of participation in the EU $6^{\text {th }}$ Framework Network of excellence "Cancer Control using Population-based Registries and Biobanks". The manual includes SOPs of information security, data management, handling of samples, project logistics and deviation i.e., content overview is available at www.kreftregisteret.no/janus. Validation of an established SOP is important to ensure that the proposed procedure fits the purpose. The SOPs in Janus are in accordance with NCI, OECD and ISBER guidelines (9-11).

The sample collecting and handling in the Janus Serum Bank has been fairly unchanged over time (7). The effects due to preanalytical sample handling, storage temperature $\left(-25^{\circ} \mathrm{C}\right)$ and long storage time of the samples, have been measured in stability studies of electrolytes, metabolites, enzymes, immunoglobulins, transport proteins, vitamins and hormones. Bilirubin (light sensitive) and potassium (sensitive for prolonged clot-time) were influenced by the sample handling (12). Sodium indicated about 4\% sublimation in the samples after 25 years storage. The studies further showed small or no changes for serum level of calcium, iron, creatinine, uric acid, albumin, AST, cystatin C, IgE, IgG, SHBG and transferrin after 25 years of storage. However, ferritin, ALT, CK $(12,13)$ and folate (14) were subjected for degradation or changed conformation.

Although the biobank was not established to collect genetic material, the samples contain trace amounts of DNA that enable molecular analyses within limited scope. So far the samples have been used in two genotyping studies with a success rate of up to $97.7 \%$ $(15,16)$. The novel biomarker microRNA has recently been found to be remarkably stable in long-term stored samples from the Janus repository (17). Furthermore, a number of ongoing research projects will provide answers regarding the utility of the DNA material in the various specimens.

A historical biobank as Janus will reflect time specific changes in the background population and differences in serum components between old and new samples may therefore be caused by other factors than storage conditions. For instance, relatively large 


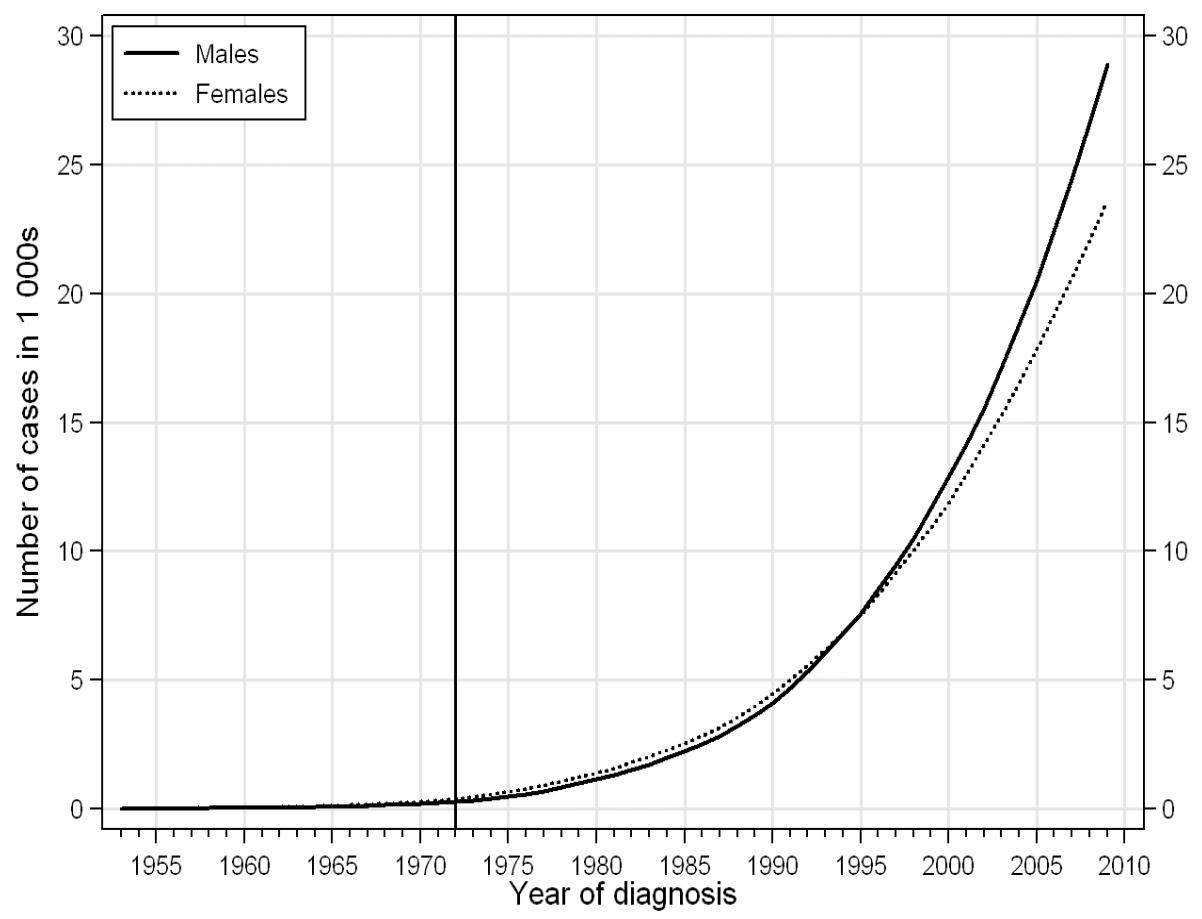

Figure 1. Number of cancer cases among Janus donors in the period 1953-2009 by year of diagnosis and sex.

changes in life style have taken place in the Norwegian population the last decades, including increased obesity, increased alcohol use, less smoking and less physical activity, that all may influence biochemical processes. This is an important aspect to consider in the interpretation of analytical results, in studies comparing samples over calendar time.

An important hallmark in the Janus Serum Bank is the unique code-keeping policy in research projects. The code of the case-control status is never revealed before the laboratory analysis is finalized, and results are returned to the Janus secretariat for long-term archiving. This procedure protects against manipulation of results and research fraud. In collaboration with Nordic biobanks we have recently published a paper focusing on important quality aspects in biobank based research, like study design, methodological approaches, matching variables, selection criteria, data linkages and logistics (18).

\section{CANCER INCIDENCE}

Once a year, the Janus cohort is linked to the Cancer Registry to identify new cancer cases among the donors. The cohort members were followed up for the event of cancer in the period 1953 to the end of 2009 . The number of cancer cases has increased year by year since 1978, the largest increase in the period 19952009 (figure 1). The number is expected to increase in the years to come as a consequence of the aging Janus cohort.

By the end of 2009 the total number of incident cancer cases with remaining blood volume in the Janus repository was 52464 . Table 1 shows the sub site specific numbers of cancer in the period 1953-2009. As shown in the table a considerable number of rare cancer sites have developed among Janus donors, for instance 179 cases of liver cancer and 660 multiple myeloma cases.

In male donors prostate cancer is the most common cancer $(n=7468)$, with a proportion of $26 \%$ of the total number of cancer cases (figure 2). The second most common cancer is lung cancer $(\mathrm{n}=3222)$, followed by colon $(n=2205)$, bladder $(n=2040)$ and melanoma of the skin $(\mathrm{n}=1663)$.

Breast cancer is the most common cancer in female donors $(n=6774)$, constituting $29 \%$ of the total number of cancer (figure 2). Cancer of the colon $(n=1707)$ is the second most common cancer followed by lung cancer $(n=1650)$ and melanoma of the skin $(n=1423)$. Cancer of the genital organs, cervix uteri, corpus uteri and ovaries combined, counts 4176 cases.

\section{RESEARCH ACTIVITIES AND SCIENTIFIC OUTCOME}

Biospecimens from the Janus repository have been utilized in a large number of studies published in international journals since the 1980ies, either in joint collaborative Nordic or European networks or as sole contributor. A complete list of publications is available at the website www.kreftregisteret.no/janus.

The scientific outcome fits well with the intended purposes. In brief; the studies have focused on i) infections and cancer, ii) biomarkers for early detection of cancer, iii) environmental exposures and cancer, iv) lifestyle related risk factors and cancer. As early as in 1997 the association between HPV and cervical cancer 
Table 1. Number of incident cancer cases in male and female donors. Follow up 1953-2009.

\begin{tabular}{|c|c|c|c|c|}
\hline ICD 10 & Site & Males & Females & Total \\
\hline C00-96 & All sites & 28894 & 23570 & 52464 \\
\hline C00-14 & Mouth, pharynx & 731 & 281 & 1012 \\
\hline $\mathrm{C} 00$ & Lip & 169 & 70 & 239 \\
\hline $\mathrm{C} 01-02$ & Tongue & 150 & 56 & 206 \\
\hline $\mathrm{C} 03-06$ & Mouth, other & 142 & 42 & 184 \\
\hline C07-08 & Salivary glands & 56 & 50 & 106 \\
\hline C09-14 & Pharynx & 214 & 63 & 277 \\
\hline C15-26 & Digestive organs & 6121 & 3874 & 9995 \\
\hline $\mathrm{C} 15$ & Oesophagus & 301 & 74 & 375 \\
\hline $\mathrm{C} 16$ & Stomach & 892 & 370 & 1262 \\
\hline $\mathrm{C} 17$ & Small intestine & 113 & 77 & 190 \\
\hline $\mathrm{C} 18$ & Colon & 2205 & 1707 & 3912 \\
\hline $\mathrm{C} 19-21$ & Rectum, rectosigmoid, anus & 1577 & 954 & 2531 \\
\hline $\mathrm{C} 22$ & Liver & 131 & 48 & 179 \\
\hline $\mathrm{C} 23-24$ & Gallbladder, bile ducts & 126 & 132 & 258 \\
\hline $\mathrm{C} 25$ & Pancreas & 698 & 459 & 1157 \\
\hline $\mathrm{C} 26$ & Other digestive organs & 78 & 53 & 131 \\
\hline C30-34, C38 & Respiratory organs & 3567 & 1734 & 5301 \\
\hline $\mathrm{C} 30-31$ & Nose, sinuses & 62 & 27 & 89 \\
\hline $\mathrm{C} 32$ & Larynx, epiglottis & 256 & 45 & 301 \\
\hline $\mathrm{C} 33-34$ & Lung, trachea & 3222 & 1650 & 4872 \\
\hline $\mathrm{C} 38$ & Mediastinum, pleura (non-mesothelioma) & 27 & 12 & 39 \\
\hline C40-41 & Bone & 42 & 44 & 86 \\
\hline $\mathbf{C 4 3}$ & Melanoma of the skin & 1663 & 1423 & 3086 \\
\hline $\mathrm{C} 44$ & Skin, non-melanoma & 983 & 478 & 1461 \\
\hline $\mathrm{C45}$ & Mesothelioma & 127 & 17 & 144 \\
\hline $\mathrm{C} 46$ & Kaposi's sarcoma & 9 & 3 & 12 \\
\hline $\mathrm{C} 47$ & Autonomic nervous system & 16 & 10 & 26 \\
\hline C48-49 & Soft tissues & 134 & 169 & 303 \\
\hline C50 & Breast & 34 & 6774 & 6808 \\
\hline C51-58 & Female genital organs & - & 4176 & 4176 \\
\hline $\mathrm{C} 53$ & Cervix uteri & - & 1025 & 1025 \\
\hline C54 & Corpus uteri & - & 1614 & 1614 \\
\hline $\mathrm{C} 55$ & Uterus, other & - & 12 & 12 \\
\hline C56 & Ovary & - & 1282 & 1282 \\
\hline C51-52, C57 & Other female genital & - & 13 & 13 \\
\hline C58 & Placenta & - & 230 & 230 \\
\hline C60-63 & Male genital organs & 8050 & - & 8050 \\
\hline C61 & Prostate & 7468 & - & 7468 \\
\hline C62 & Testis & 479 & - & 479 \\
\hline $\mathrm{C} 60, \mathrm{C} 63$ & Other male genital & 103 & - & 103 \\
\hline C64-68 & Urinary organs & 3003 & 965 & 3968 \\
\hline C64 & Kidney excl. renal pelvis & 866 & 407 & 1273 \\
\hline C65 & Renal pelvis & 97 & 49 & 146 \\
\hline C66-68 & Bladder, ureter, urethra & 2040 & 509 & 2549 \\
\hline C69 & Eye & 92 & 78 & 170 \\
\hline C70-72, D42-43 & Central nervous system & 947 & 989 & 1936 \\
\hline $\mathbf{C 7 3}$ & Thyroid gland & 207 & 513 & 720 \\
\hline C37, C74-75 & Other endocrine glands & 234 & 186 & 420 \\
\hline C39, C76, C80 & Other or unspecified & 459 & 349 & 808 \\
\hline C81-96, D45-47 & Lymphoid and haematopoietic tissue & 2475 & 1507 & 3982 \\
\hline $\mathrm{C} 81$ & Hodgkin lymphoma & 140 & 108 & 248 \\
\hline C82-85, C96 & Non-Hodgkin lymphoma & 1104 & 712 & 1816 \\
\hline $\mathrm{C} 88$ & Malignant immunoproliferative diseases & 61 & 20 & 81 \\
\hline $\mathrm{C} 90$ & Multiple myeloma & 426 & 234 & 660 \\
\hline C91-95, D45-47 & Leukaemia & 744 & 433 & 1177 \\
\hline
\end{tabular}




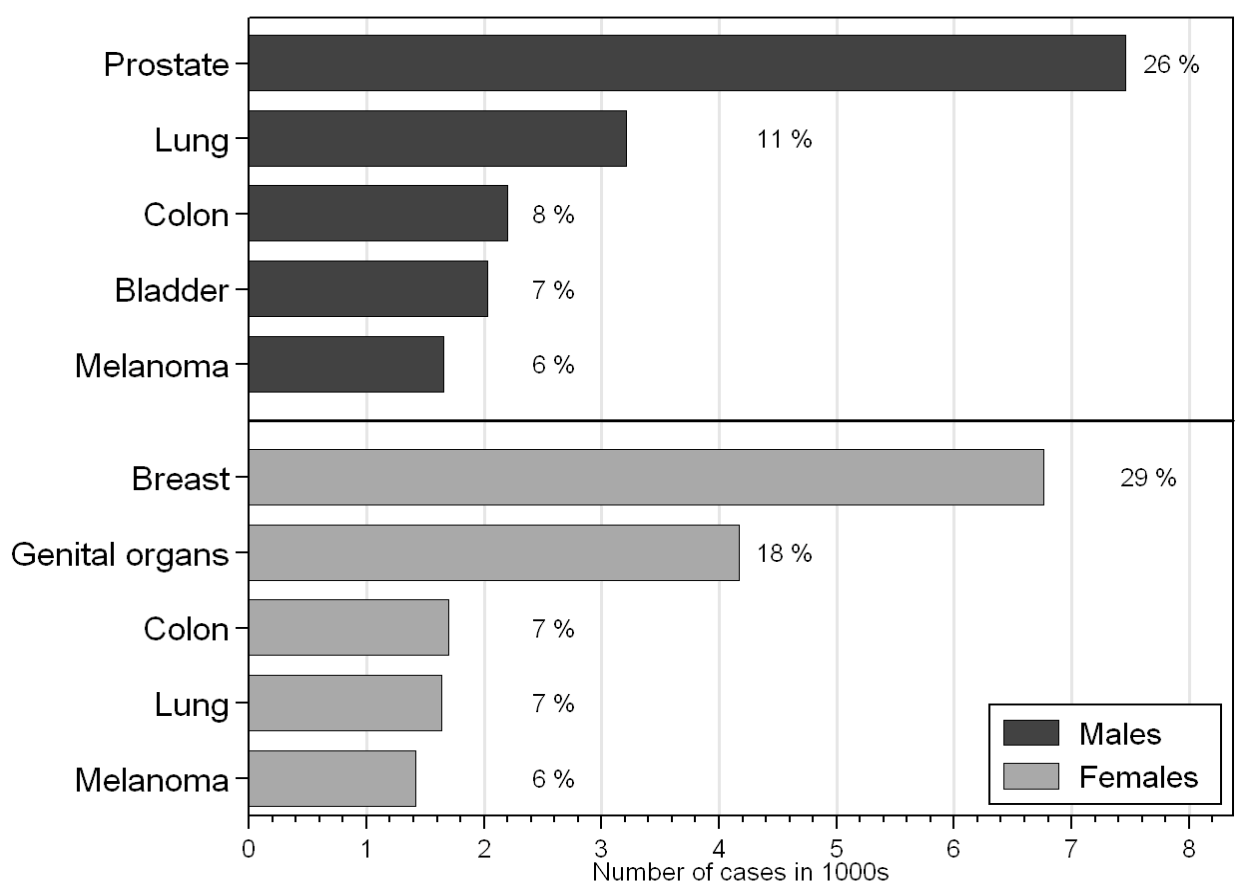

Figure 2. Proportion of common cancer cases among male and female Janus donors.

was found (19). Later, HPV was found to be positively associated with other types of cancers i.e. non-cervical anogential (20) esophageal (21) and head and neck (22). Another early observation from Janus was that Helicobacter pylori could cause gastric cancer (23).

Access to biological samples combined with data from population-based cancer registries and census occupational data, in studies of work-related and environmental exposure, is needed (24). Such studies are possible to perform by using specimens and data from the Janus Serum Bank. Research on biomarkers of environmental exposures is an on-going research topic, e.g. studies on organochlorine exposures and cancer has been performed in collaboration with the National Cancer Institute USA (25-27).

In recent years the applications for access to samples have been more oriented towards molecular epidemiological studies, microRNA profiling in cancer and omics-based studies. There is also an increasing interest in networking projects for data harmonization and sharing. Most recently the Janus cohort was involved in a large consortium of agricultural cohorts (28). A growing trend is also seen in the optimalization of biobanking by reuse of analytical data, published years ago, in new meta-analyses (29).

\section{REMARKS AND FUTURE PERSPECTIVES}

The Janus Serum Bank has been and will continue to be an important contributor to international research on biobanking and cancer research. Looking back in time the mode of operation is, in many respects, an example for high quality biobanking. Solutions for several of the current problems were, with incredible foresight, established decades ago. The large number of publications in high impact scientific journals was achieved thanks to efforts of the long-standing highly qualified Janus steering board, which has regulated the access to biospecimens in all research projects. A scientific steering board is indeed recommended and should be mandatory in all biobank operations.

The repository comprises samples stored over a long period of time and presents the opportunity to investigate accumulated, prospectively occurred cancer cases. The sample accessibility to a large number of healthy control individuals make it very timely to set up large studies with adequate statistical power to demonstrate contrasts in exposure between cancer cases and healthy controls, and to address the long-term effects of past exposure profiles, individual susceptibility and timelag effects.

An important future aim is to communicate the documentation of specimen quality for improved study quality when using samples from an historical biobank. To achieve this a number of stability studies will be performed using samples from different periods of the collection, in the same way as in modern biobank operation $(30,31)$. Of specific interest is the investigation of the quality and amount of DNA-material in the Janus repository. Further, the intention is to utilise modern technology to enable the use of the specimens in gene-environment-interaction and omics-based studies.

The most important future goal for the Janus Serum Bank is to promote excellence in the science of biobanking, ensuring that the repository is optimally used for its designed purpose - contribute to progress in cancer research. 


\section{ACKNOWLEDGEMENT}

We express our gratitude to all persons that have donated blood to the Janus Serum Bank and to all enthusiasts that made the sample collection possible. We thank the Norwe- gian Cancer Society for financial support through all the years of sample collection and the Cancer Registry of Norway for data management and responsible of regulation and licenses. We further thank the Janus steering board for the long standing scientific evaluation of research protocols.

\section{REFERENCES}

1. Moore HM, Compton CC, Lim MD, Vaught J, Christiansen KN, Alper J. Biospecimen research network symposium: advancing cancer research through biospecimen science. Cancer Res 2009; 69 (17): 6770-2.

2. Moore HM, Kelly AB, Jewell SD, McShane LM, Clark DP, Greenspan R, et al. Biospecimen reporting for improved study quality (BRISQ). Cancer Cytopathol 2011; 119 (2): 92-102.

3. Vineis P, Perera F. Molecular epidemiology and biomarkers in etiologic cancer research: the new in light of the old. Cancer Epidemiol Biomarkers Prev 2007; 16 (10): 1954-65.

4. Torgersen O. [The Janus project]. Tidsskr Nor Lageforen 1975; 95 (28): 1611-3.

5. Jellum E, Andersen A, Lund-Larsen P, Theodorsen L, Orjasaeter H. The JANUS serum bank. Sci Total Environ 1993; 139-140: 527-35.

6. Jellum E, Andersen A, Lund-Larsen P, Theodorsen L, Orjasaeter H. Experiences of the Janus Serum Bank in Norway. Environ Health Perspect 1995; 103 (Suppl 3): 85-8.

7. Langseth H, Gislefoss RE, Martinsen JI, et al. The Janus Serum Bank - From sample collection to cancer research. Oslo: Cancer Registry of Norway, 2009. http://www.kreftregisteret.no/Global/Publikasjoner\%20 og\%20rapporter/CIN_2008_Special_Issue_Janus_web.pdf.

8. Elliott P, Peakman TC. The UK Biobank sample handling and storage protocol for the collection, processing and archiving of human blood and urine. Int J Epidemiol 2008; 37 (2): 234-44.

9. National Cancer Institute, 2011. http://biospecimens.cancer.gov/bestpractices/2011-NCIBestPractices.pdf.

10. OECD, 2009. http://www.oecd.org/dataoecd/41/47/44054609.pdf.

11. International Society for Biological and Environmental Repositories. ISBER 2008 http://www.isber.org/ bp/BestPractices2008.pdf.

12. Gislefoss RE, Grimsrud TK, Morkrid L. Long-term stability of serum components in the Janus Serum Bank. Scand J Clin Lab Invest 2008; 68 (5): 402-9.

13. Gislefoss RE, Grimsrud TK, Morkrid L. Stability of selected serum proteins after long-term storage in the Janus Serum Bank. Clin Chem Lab Med 2009; 47 (5): 596-603.

14. Hannisdal R, Gislefoss RE, Grimsrud TK, Hustad S, Morkrid L, Ueland PM. Analytical recovery of folate and its degradation products in human serum stored at -25 degrees C for up to 29 years. $J$ Nutr 2010; 140 (3): 522-6.

15. Bjorge T, Lie AK, Hovig E, Gislefoss RE, Hansen S, Jellum E, et al. BRCA1 mutations in ovarian cancer and borderline tumours in Norway: a nested case-control study. Br J Cancer 2004; 91 (10): 1829-34.

16. Ulvik A, Vollset SE, Hansen S, Gislefoss R, Jellum E, Ueland PM. Colorectal cancer and the methylenetetrahydrofolate reductase $677 \mathrm{C}->\mathrm{T}$ and methionine synthase $2756 \mathrm{~A}->\mathrm{G}$ polymorphisms: a study of 2,168 case-control pairs from the JANUS cohort. Cancer Epidemiol Biomarkers Prev 2004; 13 (12): 2175-80.

17. Keller A, Leidinger P, Gislefoss R, Haugen Aa, Langseth H, Staehler P, et al. Stable serum miRNA profiles as potential tool for non-invasive lung cancer diagnosis. RNA Biology 2011; 8 (3): 1-11.

18. Langseth H, Luostarinen T, Bray F, Dillner J. Ensuring quality in studies linking cancer registries and biobanks. Acta Oncol 2010; 49 (3): 368-77.

19. Dillner J, Lehtinen M, Bjorge T, Luostarinen T, Youngman L, Jellum E, et al. Prospective seroepidemiologic study of human papillomavirus infection as a risk factor for invasive cervical cancer. J Natl Cancer Inst 1997; 89 (17): 1293-9.

20. Bjorge T, Dillner J, Anttila T, Engeland A, Hakulinen T, Jellum E, et al. Prospective seroepidemiological study of role of human papillomavirus in non-cervical anogenital cancers. BMJ 1997; 315 (7109): 646-9.

21. Bjorge T, Hakulinen T, Engeland A, Jellum E, Koskela P, Lehtinen M, et al. A prospective, seroepidemiological study of the role of human papillomavirus in esophageal cancer in Norway. Cancer Res 1997; 57 (18): 3989-92.

22. Mork J, Lie AK, Glattre E, Hallmans G, Jellum E, Koskela P, et al. Human papillomavirus infection as a risk factor for squamous-cell carcinoma of the head and neck. N Engl J Med 2001; 344 (15): 1125-31.

23. Parsonnet J, Hansen S, Rodriguez L, Gelb AB, Warnke RA, Jellum E, et al. Helicobacter pylori infection and gastric lymphoma. N Engl J Med 1994; 330 (18): 1267-71.

24. Ward EM, Schulte PA, Straif K, Hopf NB, Caldwell JC, Carreon T, et al. Research recommendations for selected IARC-classified agents. Environ Health Perspect 2010; 118 (10): 1355-62. 
25. Ward EM, Schulte P, Grajewski B, Andersen A, Patterson DG, Jr., Turner W, et al. Serum organochlorine levels and breast cancer: a nested case-control study of Norwegian women. Cancer Epidemiol Biomarkers Prev 2000; 9 (12): 1357-67.

26. Engel LS, Laden F, Andersen A, Strickland PT, Blair A, Needham LL, et al. Polychlorinated biphenyl levels in peripheral blood and non-Hodgkin's lymphoma: a report from three cohorts. Cancer Res 2007; 67 (11): $5545-52$

27. Purdue MP, Engel LS, Langseth H, Needham LL, Andersen A, Barr DB, et al. Prediagnostic serum concentrations of organochlorine compounds and risk of testicular germ cell tumors. Environ Health Perspect 2009; 117 (10): 1514-9.

28. Leon ME, Beane Freeman LE, Douwes J, Hoppin JA, Kromhout H, Lebailly P, et al. AGRICOH: A Consortium of Agricultural Cohorts. Int J Environ Res Public Health 2011; 8 (5): 1341-57.

29. Roddam AW, Allen NE, Appleby P, Key TJ. Endogenous sex hormones and prostate cancer: a collaborative analysis of 18 prospective studies. $J$ Natl Cancer Inst 2008; 100 (3): 170-83.

30. Moore HM, Kelly AB, Jewell SD, McShane LM, Clark DP, Greenspan R, et al. Biospecimen reporting for improved study quality (BRISQ). Cancer Cytopathol 2011; 119 (2): 92-102.

31. Vaught J, Rogers J, Myers K, Lim MD, Lockhart N, Moore H, et al. An NCI perspective on creating sustainable biospecimen resources. J Natl Cancer Inst Monogr 2011; 2011 (42): 1-7. 